\title{
Écrire pour prononcer son nom
}

\section{(Gwenaëlle Aubry, Personne)}

Writing to Say His Name. Gwenaëlle Aubry, Personne

\section{Claude Burgelin}

\section{OpenEdition \\ Journals}

Édition électronique

URL : https://journals.openedition.org/recherchestravaux/2886

DOI : 10.4000/recherchestravaux.2886

ISSN : 1969-6434

Éditeur

UGA Éditions/Université Grenoble Alpes

Édition imprimée

ISBN : 978-2-37747-241-3

ISSN : 0151-1874

\section{Référence électronique}

Claude Burgelin, «Écrire pour prononcer son nom », Recherches \& Travaux [En ligne], 97 | 2020, mis en ligne le 12 novembre 2020, consulté le 02 juillet 2021. URL : http://journals.openedition.org/ recherchestravaux/2886; DOI : https://doi.org/10.4000/recherchestravaux.2886

Ce document a été généré automatiquement le 2 juillet 2021.

(c) Recherches \& Travaux 


\title{
Écrire pour prononcer son nom
}

\author{
(Gwenaëlle Aubry, Personne) \\ Writing to Say His Name. Gwenaëlle Aubry, Personne
}

\section{Claude Burgelin}

1 Personne est le portrait, en vingt-six angles et au centre absent, en vingt-six autres et au moi échappé, d'un mélancolique. Lettre après lettre, ce roman-abécédaire recompose la figure d'un disparu qui, de son vivant déjà, était étranger au monde et à lui-même. [...] Personne, comme le nom de l'absence, personne comme l'identité d'un homme qui, pour n'avoir jamais fait bloc avec lui-même, a laissé place à tous les autres en lui ${ }^{1}$.

2 Malgré l'étiquette proposée, Personne n'a rien d'un roman. Il s'agit d'une sorte de travail d'orfèvrerie qui « recompose » avec le plus de ramifications ou d'étoilements possibles qui fut le père de la narratrice et qui il fut pour elle. Pas de roman, donc, mais un « je » qui cherche les vérités d'un être et la portée musicale où l'inscrire. Ce qui fait la force de ce texte, c'est la quête de la note juste, l'exigence intérieure qui gouverne le propos. Il obéit à une double polarité : éclairer ou simplement décrire quelques-uns des brouillards dans lesquels s'est perdu François-Xavier Aubry, professeur de droit à l'université Panthéon-Sorbonne, né en 1945, mort en 2006, ayant basculé dans la maladie mentale (psychose maniaco-dépressive si l'on tient aux catégorisations) et diverses formes de clochardisation ou de lâcher-prise. L'autre polarité demeure davantage à l'arrière-fond, mais oriente tout autant le tourbillon des interrogations de sa fille : quelle place donner, dans ce qu'elle est devenue, dans ce qui l'a construite, à cet être de dérive, d'ombre et d'opacité ? Comment trouver le plus intime degré de l'intellection et de l'empathie tout en gardant la distance nécessaire pour l'exercice de la justesse? Trajectoire littéraire autant que psycho-mentale d'une grande rigueur: donner à entrevoir le pathétique de la vie de ce père et ce que signifiait d'être la fille de cet homme en perdition, sans laisser un pouce de terrain au pathos.

3 Gwenaëlle Aubry est philosophe, universitaire comme son père. Elle est spécialiste de Plotin, un philosophe qui a construit sa pensée autour des relations de l'âme et du corps. Outre ses travaux sur Plotin, elle a écrit des romans dont l'un, L'Isolée (2002), suivi de L'Isolement (2003), s'inspire de l'histoire de Florence Rey (fille d'un père 
schizophrène) et d'Audry Maupin, ce couple d'étudiants dont la dérive meurtrière a en quelque sorte signé la fin d'« Action directe » (1994). François-Xavier Aubry a été un spécialiste des questions de droit liées à la décentralisation. On ne l'aurait pas inventé tant la dislocation, la perte du lien central, a été le symptôme majeur de cet homme. Il a été marié quelques années, a eu deux filles. Quand l'aînée, Gwenaëlle, a cinq ans, le couple divorce. Le père devient un père de week-end et d'un mois de vacances.

Personne se présente éclaté en vingt-six fragments. Le titre de chacun de ces chapitres est tributaire de l'ordre alphabétique, de A comme « Antonin Artaud » pour le premier, à $\mathrm{Z}$ comme "Zelig " pour le dernier. Une façon de présenter un désordre sous une forme ordonnée, celle peu contraignante de la suite alphabétique. Cette fragmentation d'une histoire et d'une personne en vingt-six pièces de puzzle laisse au lecteur cette tâche passionnante autant qu'impossible : tenter d'assembler ces morceaux tout en les laissant épars.

5 Gwenaëlle Aubry s'est écartée de l'ordre chronologique pour "recomposer » un portrait décomposé de son père. Signalant ainsi qu'elle n'essayait pas de retracer la genèse de la folie de cet homme. Nous sont mis sous les yeux les aboutissants de cet éclatement mental, mais non les tenants. Il y a là un moment neuf du travail biographique, très en retrait de l'approche psychanalytique et de la nosographie psychiatrique. L'écriture se veut «traitre » « aux voix qui disent “je" sans trembler [...] aux récits linéaires, aux alphabets familiers/aux vies encadrées par deux dates et qui de l'une à l'autre se déploient sans renaissances ni disparitions » ( $P$, p. 143).

On peut penser, lisant ce livre, à la façon dont Georges Perec met en valeur la méthode de Robert Antelme :

Dans L'Espèce humaine, le camp n'est jamais donné. Il s'impose, il émerge lentement.

[...] Il n'est pas images toutes faites, et rassurantes dans leur violence même. [...] Il n'y a pas d'explications. Mais il n'est pas un fait qui ne se dépasse, ne se transforme, ne s'intègre à une perspective plus vaste. [...] Il n'est pas un fait qui ne devienne exemplaire. Le récit s'interrompt à chaque instant, la conscience s'infiltre dans l'anecdote, l'épaissit [...] puis débouche sur un autre souvenir ${ }^{2}$.

7 C'est donc une mosaïque que dessine la narratrice. Les différentes facettes de la folie de François-Xavier Aubry "s'imposent ", "émergent lentement », pour reprendre les mots de Perec. C'est d'abord de la singularité de cette histoire qu'il y a à rendre compte. Cette " décentralisation ", qui est la maladie ou la faille de ce père, devient au contraire méthode heuristique. On ne peut approcher pareille difficulté d'être que par des coupes, des épaississements, des raccords, par la mise en place «d'un certain type de relations entre les éléments du récit, hiérarchisation, intégration, progression ", brisant " l'image immédiate et inopérante ${ }^{3}$ » qu'on se fait de la maladie mentale. Il est d'ailleurs à noter l'usage prudent que fait Gwenaëlle Aubry du mot « maladie ».

8 Autrement dit, il s'agit d'un texte tout en va-et-vient, en trouées, en échappées et en retours amont, qui suppose de la part du lecteur vigilance, liberté mentale, ductilité... Les lieux ne sont que discrètement marqués (la « ville de province »). Peu de dates sont indiquées. Ces précisions restent en arrière-scène. Il ne s'agit pas d'un récit, d'une histoire, mais d'une suite entrecoupée de cristallisations ou d'instants choisis pour leur pouvoir d'évocation ou d'intellection.

9 Il ne nous est pas vraiment donné de suivre les étapes du dysfonctionnement de François-Xavier Aubry, d'autant moins faciles à saisir qu'elles s'effacent en une suite de stop and go, de dégradations, d'améliorations, de stabilisations, de rechutes. La maladie 
se présente comme une succession de fluctuations, de dérives en flux et reflux. On ne trouve donc guère de moments de mutation décisive, de prises de conscience tranchées. "Je ne sais pas quand je me suis dit pour la première fois "mon père est fou", quand j'ai adopté ce mot de folie, ce mot emphatique, vague, inquiétant et légèrement exaltant, qui ne nommait rien, en fait, rien d'autre que mon angoisse. » $(P$, p. 25) Le temps, à la fois continu et discontinu, de la maladie mentale en appelait à une narration elle aussi faite d'entrelacements des lignes de fuite et des lignes de suite.

Il s'agit en quelque sorte d'une phénoménologie très ondoyante et légère (donnant par là même d'autant plus de poids aux formules tranchantes). Le discours sur la maladie mentale est souvent... du discours, lourd et mettant bien des barrières pour s'interdire toute forme de proximité. Et le lexique du diagnostic sert à accentuer cette distance. Ce que Gwenaëlle Aubry cherche à tracer, c'est l'étrange, l'imprévisible aventure de la pensée et du sentiment vécue par la victime comme par le témoin navré de cette trajectoire devenue invivable. Elle esquive les représentations toutes faites sur la genèse et les manifestations de la psychose. Elle ne les récuse pas, mais ne fait que les croiser ou les traverser en dissolvant le caillot qui obture la liberté de penser et de ressentir que ces mots imposent. Le fantôme de la pensée de Foucault circule là, de façon aérée et personnelle, sans que son nom apparaisse.

11 Ce que permet cette mise en constellation de vingt-six moments éclairants, c'est une très stimulante fluctuation dans les distances. On voit Gwenaëlle (ce prénom n'est jamais prononcé) petite fille, adolescente, jeune fille, jeune mère cherchant sa place auprès de/loin de ce père essentiellement défaillant. Passant de l'enfance à l'âge adulte, elle ne cesse de changer de regards sur cet homme qui lui-même oscille dans les manières de considérer son mal. Rien ne semble plus rigide ou monotone qu'une structure délirante. Or ici, c'est à une suite de métamorphoses ou du moins de changements marqués qu'on assiste chez cet homme - et donc chez celle qui enregistre et subit ces alternances entre immobilisations et excessives mobilités. Il y a là quelque chose d'à la fois romanesque et d'intellectuellement troublant.

Une des forces du livre est d'éviter l'univers du procès (comme aussi celui de la plainte). La colère émerge parfois, mais est esquivé le réquisitoire contre un tel père ou contre les institutions qui l'ont pris en charge. Ce qui a rendu cet homme délirant n'est pas abordé frontalement. Demeurent son mystère, la singularité de ses conduites plus ou moins opaques. Si procès il y a, mais là n'est pas le propos, c'est celui fait aux familles, surtout celle du père et des siens. Là encore le procès est dressé autant contre les humains que contre les codes qui les meuvent et les enchaînent. On pourra retenir cette parfaite définition d'une certaine bourgeoisie : « on était bourgeois sans haine des prolétaires, catholique sans foi, nanti sans avidité, lettré sans curiosité. » $(P$, p. 31) Et la folie est-elle peut-être d'abord celle de cette caste ligotée dans les liens du comme si et de la fausse normalité meurtrière, celle qu'évoque Fritz Zorn à propos de ses parents dans Mars, celle peut-être qui a contribué à enfoncer dans la mélancolie et l'égarement François-Xavier Aubry.

13 Le texte s'abstient de toute complaisance pour le dégradant ou le délirant dont on sait les pouvoirs de fascination. La narratrice ne cherche pas à s'aventurer du côté de ce qu'il pourrait y avoir de sadique ou de trouble dans les conduites de cet homme. Elle indique, sans plus, en staccatos légers, qu'il peut être en proie à une quête de l'humiliation (mystique? masochiste?). Mais, fondamentalement, il n'y a pas 
d'idéologisation, de thèse à soutenir concernant la folie de ce père. Son naufrage reste celui d'un homme qui a perdu le gouvernail de son navire.

Dans le chapitre "Clown» sont «impli-cités» de menus fragments du poème d'Henri Michaux qui porte ce titre ${ }^{4}$. Voici la fin de cet admirable texte :

À coups de ridicules, de déchéances (qu'est-ce que la déchéance ?), par éclatement, par vide, par une totale dissipation-dérision-purgation, j'expulserai de moi la forme qu'on croyait si bien attachée, composée, coordonnée, assortie à mon entourage et à mes semblables, si dignes, si dignes, mes semblables.

Réduit à une humilité de catastrophe, à un nivellement parfait comme après une intense trouille.

Ramené au-dessus de toute mesure à mon rang réel, au rang infime que je ne sais quelle idée-ambition m'avait fait déserter.

Anéanti quant à la hauteur, quant à l'estime.

Perdu en un endroit lointain (ou même pas), sans nom, sans identité.

CLOWN, abattant dans la risée, dans l'esclaffement, dans le grotesque, le sens que contre toute lumière je m'étais fait de mon importance.

Je plongerai.

Sans bourse dans l'infini-esprit sous-jacent ouvert à tous,

ouvert moi-même à une nouvelle et incroyable rosée

à force d'être nul

et ras,...

et risible ${ }^{5}$..

Le drame de François-Xavier Aubry serait de ne pouvoir arriver à passer de la risée à la rosée, de l'annulation à l'ouverture. Encore que... Paradoxe passionnant qu'il y a dans l'idée ou le mythe de "ne pas faire bloc avec soi-même ». Parfois, cet éclatement mène à cette qualité de centration à laquelle n'aboutissent que les saints, les sages version orientale, les jusqu'au-boutistes du dépouillement, parvenant à défaire la citadelle du moi. Ce destin, François-Xavier Aubry semble s'en approcher : «il a eu en partage une expérience rare et précieuse » en ayant "connu la douleur et la joie» d'une forme d'« extase ", venant " très près de ce dont portent témoignage quelques livres noirs et lumineux. » Mais « la grâce de cette dépossession » $(P, \mathrm{p} .124)$ reste éphémère. Le plus souvent, la fille représente son père comme perdu dans le bazar trop bien achalandé chez lui des identifications. Cherchant des masques pour masquer qu'il n'y aurait personne sous ces masques. Tandis que se détache celui du professeur de droit, homme d'ordre et de rigueur, seraient essayées des imagos héroïques, corsaire, espion (James Bond...), cavalier sur un cheval blanc. Ou d'autres, plus orgueilleusement masochistes comme le mouton noir. D'autres carrément bouffonnes, anarchistes, insolentes, libertaires comme le clown. Le professeur garde en son tiroir un nez rouge façon Coluche. Il allait « éternel enfant de cinq ans jonglant avec les possibles, prenant devant son miroir les poses des vies rêvées, cherchant celle qui, enfin, collerait à sa peau, s'imprimerait sur ses traits, celle dans laquelle sa foule intérieure pourrait se rassembler, dire d'une seule voix c'est moi. » Trop nombreux sont ceux qui « logent sous sa peau ", "qui, à travers lui, tour à tour, disaient je, qualités sans homme, attributs sans loi, atomes pulvérisés autour d'un centre absent.» $(P$, p. 120-121) Mais demeure, farouche, insistante, cette quête, cette insatisfaction éperdue, cette recherche de quelque ailleurs, de quelque absolu : dans la dégradation même, subsistent les traces de la rigueur exigeante d'un refus et de «la sorte de courage qu'il faut pour être rien et rien que rien ${ }^{6} »$.

Toutes ces identifications coexistent ou, en tout cas, ne s'annulent pas les unes les autres. Le clochard alcoolisé n'efface pas le gaulliste homme d'ordre. À glisser d'une 
identification à une autre, le voici en proie à une pluralité d'affects, entre rêveries mégalomanes et passages par l'effondrement. Cette configuration mouvante des humeurs et des instants - celle du père comme de sa fille cherchant à le cerner et à se situer au fil des âges par rapport à lui - donne au livre son tempo (faudrait-il dire son andante, voire son allegro ?). On sent la nécessité d'une architecture narrative qui donne une place fondatrice aux ruptures. Mais le lecteur est au fil des chapitres porté par des phrases assez longues, se relançant elles-mêmes par des suites de juxtapositions, dans une respiration aisée de la syntaxe et des parataxes. Le propos se ramifie, avec des embranchements qui visent autant au buissonnement du sens qu'à l'ouverture vers d'autres directions. On sent à l'œuvre une écriture qui se garde de ce qui délimite ou arrête trop tôt les impressions ou le mouvement de la pensée.

Souvent sont associées des notions de portée différente, laissant du jeu entre elles. Question de doigté ou d'exigence. Comme dans l'évocation de «tout ce que nos vies mêlées ont déposé en moi, silencieusement / brisures, éclats, décombres / tendresses, désirs et choix. " $(P$, p. 144) Tournant autour de ce qu'impliquait dire "mon père ", apparaît «cet écart infranchissable entre les mots des autres et mon langage privé : "mon père", c'est-à-dire mon délire, ma détresse, mon dément, mon différent, mon deuil, mon disparu. » ( $P$, p. 38) Deux mots reviennent : ombre, d'une si grande richesse sémantique, et peut-être laissant l'affirmation se nuancer, s'ouvrir, risquer une échappée.

Artaud, Michaux, Perec (appelé par quelques mots de $W$ ou le souvenir d'enfance) ... C'est grâce à la littérature que peuvent être dites, avec leurs résonances, les déconnexions du père et les oscillations de sa fille prenant appui sur les mots et les images de tel ou tel, à peine un collage, un effleurement, une mise sur rails. Gwenaëlle Aubry se souvient-elle de La Vie mode d'emploi lorsqu'elle dit de son père : «[...] ce qu'il y avait dans ses yeux, ce n'était pas de la peur, ce n'était pas de la détresse, ce n'était pas du vide non plus, mais une absolue nudité » $(P$, p. 35$)$ ? Alors que Perec évoque ainsi Bartlebooth : « Il y avait dans ce regard qui l'évitait quelque chose de beaucoup plus violent que le vide, quelque chose qui n'était pas seulement de l'orgueil ou de la haine, mais presque de la panique, quelque chose comme un espoir insensé, comme un appel au secours, comme un signal de détresse ${ }^{7}$.»

Parlant d'écriture, on touche au lien secret, jamais exposé ou explicité, mais évident à la lecture, entre ce père et sa fille. Lui lit les travaux de sa philosophe de fille sur Plotin et les commente à sa façon en l'appelant « l'auteur ». Si l'écrivaine Gwenaëlle Aubry est magnifiquement inspirée, elle a de qui tenir : son père était remarquablement doué. Il a beaucoup tenu de journaux. Et impressionnent sa fermeté de pensée, la qualité de frappe de ses phrases, souvent sa lucidité sur son mal, son acuité, parfois son humour. Dans le dérapage ou le délirant, le propos garde une belle tenue. Le professeur de droit était un homme à l'imagination nette et au verbe superbe (on le voit notamment dans de très beaux récits de rêves). Il est difficile de ne pas penser à Nerval en certains moments. Voici quelques exemples de la prose de François-Xavier Aubry :

Je me souviens de la terrasse ensoleillée du dernier hôpital, où j'ai mesuré que je ne pouvais pas en vouloir à Dieu de la nouvelle destinée qui s'annonçait pour moi épaulé par mes filles. Certains étaient vraiment seuls comme cette pauvre grosse énurésique qui ne comptait même plus les années passées en internement, ce grand homme qui s'était immolé par le feu et dont le visage était comme fondu, toujours sans visites et toujours d'une grande affabilité, une vieille dame habillée comme une petite fille avec un chapeau de paille à la Renoir, une ancienne danseuse de 
l'opéra devenue schizophrène, sans oublier tous ces petits vieux que l'on dit fous et que seule leur inutilité sociale condamne, et les SDF. Dans cet hôpital, j'ai beaucoup, beaucoup dialogué, la parole m'étant revenue sous la voûte d'arbres splendides. $(P$, p. 63-64)

Il m'est souvent arrivé de me rendre dans les salons de grands hôtels parisiens pour regarder les hommes d'affaires arabes dans l'espoir que l'un d'entre eux me ramène là-bas, dans la blancheur du sable. $(P$, p. 84)

Il faut, à l'être fragilisé qui sait qu'il ne sera jamais le héros de Goethe, le surhomme de Nietzsche, protéger son retour à la vie en cherchant l'unité de son âme, dissociée et imparfaite, et en soignant en lui l'animal blessé. ( $P$, p. 98)

Père et fille communient dans la passion de l'écriture, de la noblesse des phrases, le besoin des mots qui discernent pour mettre en perspective et à distance la déréliction. On se prend à rêver à ce que pourrait être un montage des propos de FrançoisXavier Aubry qui dessinerait le paysage singulier de son monde et de ses arrière-fonds. L'écriture semble, à partir des quelques fragments qui nous sont livrés, un lieu où se rassemblent ces fils qui paraissent lui échapper. Elle lui permet d'ouvrir des fenêtres (vers? sur?) dans un édifice verbal qui a structure et architecture. Sur la scène de l'écriture, il n'est pas "personne »: celle-ci témoigne d'un homme vivant et pensant dans ses effondrements mêmes. Il a, à un moment, composé un texte en deux parties, «L'Épreuve » et «L'Espoir » : écrivant, dit sa fille au terme du chapitre «Pirate », «ces pages qu'à mon tour je tente de déchiffrer, dont j'essaie d'entendre le langage, cherchant des repères dans mon alphabet familier, des résonances dans le passé qu'il a romancé, un ordre et un sens dans le chaos partagé, prenant appui sur ses mots, ses phrases et sa mémoire, sans plus voir, à force, si ce que je pirate ainsi est sagesse ou folie.» ( $P$, p. 119) En-deçà de cette sagesse peut-être folie, il y a, chez tous deux, cette confiance dans la langue, comme si la tenue des phrases, la musicalité, le rythme des cadences étaient la plus sûre des rambardes.

Quelle est, au bout du compte, la nature de ce texte ? Un "tombeau», un tombeau de mots remplaçant la dalle absente? François-Xavier Aubry avait « refusé la tombe, la pierre, le masque de gisant et l'ultime visage, il a préféré les cendres à tout vent dispersées $^{8}$, peut-être a-t-il trouvé, dans le désert blanc de la mort, ce que depuis toujours il cherchait : le droit, enfin, de ne plus être quelqu'un ${ }^{9}$ ?» ( $P$, p. 159) Mais il y a quelque chose de pesant en ce mot de tombeau. Gwenaëlle Aubry n'est pas Antigone. La fille de ce mort est bien trop présente et vivante en ce livre où justement elle évite de s'enclore dans le destin de ce père. Seule l'advenue de certaines images, de certains mots peut donner forme à ce " château » construit autour d'une absence :

À présent qu'il est mort, on réunit ce qu'il a laissé, miettes et cailloux semés dans les forêts de son angoisse, trésors et épaves, on construit le vide, on sculpte l'absence, on cherche une forme pour ce qui, en nous, demeure de lui, et qui a toujours été la tentation de l'informe, la menace du chaos, on cherche des mots pour ce qui, toujours, a été en nous la part secrète, la part muette, un corps de mots pour celui qui n'a pas de tombe, un château de présence, pour protéger son absence. (P, p. 17)

Un texte impossible à nommer pour donner un nom, restituer son nom à celui qui s'est acharné à le perdre... Dans le chapitre "Napoléon du Grand Nord», est dessinée la silhouette d'un autre François-Xavier Aubry qui, parti de Saint-Malo, vécut au $\mathrm{XIX}^{\mathrm{e}}$ siècle une vie d'aventurier au Far-West et mourut hérö̈quement lors d'un duel. Évoquant le premier Noël après la mort de son père, au milieu du trop-plein familial et du rituel dinde-cadeaux-champagne, alors qu'était feuilleté « un de ces livres idiots que l'on offre ces jours-là, un dictionnaire des noms propres ", ouvert à la lettre A, et qu'un 
des participants s'étonne du nombre d'Aubry trouvé au Québec, «c'est, ai-je dit très fort, à cause d'un explorateur fameux venu de Saint-Malo, François-Xavier Aubry, le Napoléon du Grand Nord, et le cercle de silence s'est refermé comme de l'eau stagnante sur la pierre que l'on vient d'y jeter, ils m'ont crue folle, peut-être, ou maligne [...] mais le deuil n'est pas folie ni la fidélité, les fous sont ceux qui oublient et se refusent à nommer, et font, toujours, comme si de rien n'était. » Le chapitre se clôt sur la phrase : " (Je ne fais rien d'autre, finalement, écrivant ce livre, que prononcer son nom.) » $(P$, p. 106-107)

Mais il a fallu tout un livre et vingt-six pièces de puzzle pour que soit prononcé ce nom, enfin arrimé, recollé, avec les harmoniques, les stridences et les silences qui conviennent.

Prononcer ce nom... S'il revient à sa fille de restituer l'honneur ou l'aura du nom de son père, de faire entendre la musique silencieuse qu'il y a en elle autour de ce nom, j'ai été, moi auditeur et commentateur de cette partition, dans un continuel embarras quand il s'agissait de nommer les protagonistes de cette histoire. Écrire «FrançoisXavier Aubry " ou "Gwenaëlle Aubry " n'allait pas de soi, tant ces appellations objectivent et figent. "Le père ", «la fille»: mots indiscutables, mais tout aussi gênants. J'ai le sentiment de m'immiscer dans une intimité où je n'ai pas à être. Et l'article défini est aussi épais que malencontreux. Dire "l'auteure ", « l'écrivaine ", « la narratrice » rabat le propos sur le théâtre de l'écriture. Évidemment le seul possible, mais dont on sait bien qu'il est une scène particulière qui modélise d'une certaine façon, avec les chicanes de ses phrases et ces points qu'il faut bien poser.

Certes, des milliers de lecteurs ont lu ce livre, offert à notre indiscrétion. Reste que j'exerce par mes propos de la marge une sorte de mainmise ou simplement de regard surplombant sur cette histoire. Quel que puisse être mon tact (si j'en ai eu et il est si facile d'en manquer), j'exerce là une sorte de prédation. Ou d'entrée en scène plus ou moins indue, irruption qui ne peut éventuellement se justifier que si mes mots tombent juste, ce dont je ne suis nullement assuré. Et dans cette histoire de nom, j'insère, tel le coucou squatteur de nid, mon nom. Avec quelle légitimité mettre sa loupe et ses phrases sur ce que viennent de vivre des personnes bel et bien vivantes, même si elles ont volontairement confié aux mots le pouvoir de dire, de raconter ou témoigner? La posture du critique est ici tangente à l'imposture.

Elle pourrait peut-être trouver une assise si j'allais chercher « d'où je parle ». Pourquoi une histoire d'orpheline ou de père défaillant me requiert-elle ? Où cette histoire de «folie» vient-elle me tourmenter, me séduire ou m'exciter? Etc. Cette arrièreboutique-là, l'arène critique n'a pas à la connaître. Ce qui n'est pas une raison pour que je ne veuille pas savoir comment et pourquoi celle-ci vient former/déformer mon commentaire. Mais ceci est une autre affaire. 


\section{NOTES}

1. Quatrième de couverture de Personne, Paris, Mercure de France, 2009. Les renvois à cette œuvre seront donnés entre parenthèses après les citations (abrégés en $P$ ).

2. G. Perec, "Robert Antelme ou la vérité de la littérature ", dans L.G. Une aventure des années soixante, Paris, Seuil, coll. « La Librairie du Xxe siècle », 1992, p. 95-96.

3. Ibid., p. 97.

4. Dernière vision du père à l'HP : «J'ai vu mon père ainsi, dénudé, détrôné, tombé, mon père devenu rien et rien que rien, mon père vidé de l'abcès d'être quelqu'un. » $(P$, p. 33)

5. H. Michaux, « Peintures » [1939], L'espace du dedans, Gallimard, 1966, p. 249.

6. H. Michaux, « Clown », ouvr. cité.

7. G. Perec, La Vie mode d'emploi, Paris, Hachette, 1978, p. 166.

8. La scène des obsèques se colore d'une touche inattendue et délicieusement transgressive quand on voit les deux filles, lestées des cendres de leur père, finir par en jeter une pincée derrière l'autel de Saint-Étienne du Mont et le reste dans les eaux de la Fontaine Médicis.

9. Derniers mots du livre.

\section{RÉSUMÉS}

Comment être la fille de "Personne ", d'un Nobody qui fut tout le contraire d'un Nobody? Peut-on réduire un homme à sa folie? Le père de la narratrice, professeur de droit, a été sévèrement atteint par la psychose maniaco-dépressive. Mais comment évoquer avec justesse cet homme, les ombres et les brouillards dans lesquels il s'est perdu ou trouvé ? Comment restituer quelque chose de la vérité de cet être, de ce qu'il eut de noble et d'inventif tout en plongeant dans le désastre ? Comment dire en même temps combien il a été difficile pour sa fille de trouver sa place par rapport à cet homme qui avait perdu place et repères? Comment trouver une structure narrative, des mots pour dire cette traversée d'un chaos?

How to be the daughter of "Nobody"? Can a man be reduced to his madness? The narrator's father, a law professor, was severely affected by manic-depressive psychosis. But how can we accurately evoke this man, the shadows and mists in which he was lost or found? How can the truth of this being, of what was noble and inventive about him be restored while plunging into disaster? At the same time, how can one say how difficult it was for his daughter to find her place in relation to this man who had lost his place and his bearings? How to find a narrative structure, words to express this journey through chaos?

\section{INDEX}

Mots-clés : structure narrative, absence, folie

Keywords : narrative structure, absence, madness 


\section{AUTEUR}

\section{CLAUDE BURGELIN}

Université Lyon 2

Claude Burgelin a enseigné la littérature française contemporaine à l'université Lyon 2. Il a publié notamment Georges Perec (Seuil, 1988), Les parties de dominos chez Monsieur Lefevre, Perec avec Freud, Perec contre Freud (Circé, 1996), Les Mal nommés (Seuil, 2012) et de nombreux articles sur des écrivains contemporains (en particulier dans le domaine de l'écriture personnelle). 